\title{
Semantic Web Meets Internet of Things and Web of Things
}

\author{
[2nd Edition]
}

\author{
Amelie Gyrard*, Pankesh Patel ${ }^{* *}$, Soumya Kanti Datta ${ }^{\ddagger}$, and Muhammad Intizar Ali $^{\dagger}$ \\ *Univ Lyon, MINES Saint-Etienne, CNRS, Laboratoire Hubert Curien, France; ${ }^{* *}$ ABB Corporate Research, India; \\ ${ }^{\ddagger}$ EURECOM, France; ${ }^{\dagger}$ Insight Center for Data Analytics, Ireland \\ *amelie.gyrard@emse.fr, ** pankesh.patel@in.abb.com, ${ }^{\star}$ dattas@eurecom.fr, \\ †ali.intizar@insight-centre.org
}

\begin{abstract}
An ever growing interest and wide adoption of Internet of Things (IoT) and Web technologies are unleashing a true potential of designing a broad range of high-quality consumer applications. Smart cities, smart buildings, and e-health are among various application domains which are currently benefiting and will continue to benefit from IoT and Web technologies in a foreseeable future. Similarly, semantic technologies have proven their effectiveness in various domains and a few among multiple challenges which semantic Web technologies are addressing are to (i) mitigate heterogeneity by providing semantic inter-operability, (ii) facilitate easy integration of data application, (iii) deduce and extract new knowledge to build applications providing smart solutions, and (iv) facilitate inter-operability among various data processes including representation, management and storage of data.

In this tutorial, our focus will be on the combination of Web technologies, Semantic Web, and IoT technologies and we will present to our audience that how a merger of these technologies is leading towards an evolution from IoT to Web of Things (WoT) to Semantic Web of Things. This tutorial will introduce the basics of Internet of Things, Web of Things and Semantic Web and will demonstrate tools and techniques designed to enable the rapid development of semantics-based Web of Things applications. One key aspect of this tutorial is to familiarize its audience with the open source tools designed by different semantic Web, IoT and WoT based projects and provide the audience a rich hands-on experience to use these tools and build smart applications with minimal efforts. Thus, reducing the learning curve to its maximum. We will showcase real-world use case scenarios which are designed using semantically-enabled WoT frameworks (e.g. CityPulse, FIESTA-IoT and M3).
\end{abstract}

\section{Keywords}

Internet of Things (IoT); Web of Things (WoT); Semantic Web of Things (SWoT); Data Interoperability; Ontologies

C) 2017 International World Wide Web Conference Committee (IW3C2), published under Creative Commons CC BY 4.0 License.

WWW 2017, April 3-7, 2017, Perth, Australia.

ACM 978-1-4503-4913-0/17/04.

http://dx.doi.org/10.1145/3038912.3038914

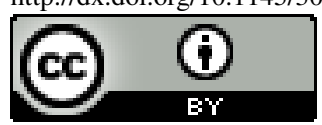

\section{MOTIVATION}

In recent years, we have been witnessing a growing number of sensors embedded in smart devices or everyday objects. Applications exploiting sensors and producing data are more and more popular. More than 100 scenarios 1 have been referenced by IoT projects such as continuous health care, smart home, smart orchard, detecting road conditions or air pollution countermeasures. However, one of the challenging problem with the existing IoT applications is device and data inter-operability. Data produced by these devices adheres to proprietary formats and use of common vocabularies is minimal. Web and its extension "Semantic web" technologies can rescue IoT technologies to overcome the challenge of inter-operability.

Through this tutorial, we aim to bridge this gap among Web technologies, IoT and WoT. We will provide our participants with an overview of the current ecosystem approaches and discuss their pros and cons. We will showcase how the limitations of existing IoT platforms can be overcome with both Web and semantic Web technologies. Moreover, we aim to provide the participants with a hands-on experience that will guide them to select a combination of right technologies for their requirements to develop their semantics-based IoT applications. The tutorial web site is available online ${ }^{2}$.

\section{AUDIENCE}

The same people attending the following (previous) workshop editions, research tracks or W3C tracks will be interested in our tutorial:

- W3C Track: Building and Designing the Web of Things

- W3C Track: Web of Things - Smart Cities, Cars And Homes

- Track: Semantics and Knowledge

- Track: Ubiquitous and Mobile Computing WWW Track

- Workshop: Linked Data on the Web (LDOW2016)

- Workshop: Web Intelligence \& Communitie: 4

- WWW Developers Day

This tutorial should be of equal interest to a wider community including Web and Semantic Web technologists, academia, students and most importantly application developers who are thriving to build IoT and WoT data based applications.

${ }_{1}^{1}$ http://www.ict-citypulse.eu/scenarios/scenarios

2 http://semantic-web-of-things . appspot.com/?p= WWW2017Tutorial

3 http://events.linkeddata.org/ldow2016/

${ }^{4}$ http://www.tmrfindia.org/WIC16/ 
Tutorial is designed for participants with all levels experience and expertise of technologies. Ideal preparation can be to have some basic know how of IoT, WoT, semantic Web and Web technologies/standards etc.

\subsection{Relevance}

Through this tutorial, our aim is to bring together the Software Engineering, Web, IoT, Semantic Web, and WoT communities together for developing future Internet applications via SWoT. Few of the learning objectives of the tutorial are the following:

- For beginners, it will create an understanding of what SWoT means, common terminology, and use cases.

- For practitioners, it will provide a solid foundation to compare and evaluate existing approaches for building SWoT applications.

- For researchers, it will provide a good discussion forum to explore open research challenges in the areas concerning all aspects of designing and developing an ecosystem for SWoT applications.

- For developers, it will demonstrate the need to design tools to assist non experts in integrating new technologies and reduce as much as possible the learning curve.

\subsection{Previous Editions}

The first successful edition of this tutorial has been conducted at International Semantic Web Conference (ISWC) 2016. More than 30 persons attended the tutorial, whereas there were 8 sessions in parallel. The program, set of slides and all materials are available onlin $£^{5}$ This is a new opportunity to disseminate our research work and expertise to a new audience and larger communities. This tutorial will be improved according to the feedback from the previous edition and will be further adapted and designed for the Web community

\subsection{Prerequisite}

Since this tutorial is for WWW 2017, we assume that the attendees have basic understanding of web technologies and programming languages. The knowledge of the Java language, prior set up of the Eclipse environment with Java 1.7 would be highly appreciated. We will provide basics to understand IoT, WoT and semantic web technologies in our presentations. Our step-by-step tutorial will guide participants to use the IoT, WoT and semantic web to develop Semantic Web of Things applications.

\subsection{Equipment}

The tutorial presenters need access to the Internet and standard equipment e.g. projector, audio system, microphone and power plugs. Participants must bring their own laptop. All relevant softwares can be downloaded from the Internet or shared through USB keys.

\section{TOPIC DESCRIPTION, TUTORIAL MA- TERIAL, DURATION \& SESSIONS}

We intend to deliver a half day tutorial. Our presentations will be mostly based on powerpoint slides, animations and

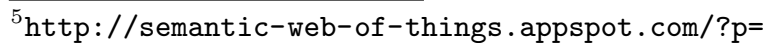
ISWC2016Tutorial small code snippets to illustrate the concepts. We will interleave live demonstration of existing systems with the presentations of the material throughout the tutorial to make it more attractive. This will provide audiences with the opportunities to study the technology in action. We will make the tutorial interactive while delivering the contents. Small and quick exercises will be asked to make the tutorial interactive and ensure that the core concepts are delivered.

\subsection{PART I: From Internet of Things (IoT) to Web of Things (WoT)}

\section{Duration: 60 mins}

Presenters: Pankesh Patel \& Soumya Kanti Datta. The tutorial will start with an introduction aimed at setting a common context between the IoT and Web for all participants. It will provide an understanding of commonly used terminology by presenting a domain model [13, 12. The entire IoT ecosystem is highly fragmented due to individualized implementations as well as data-silos arising due to non-uniform treatment of IoT data originating at heterogeneous domain. To mitigate these issues, we will leverage the IoT concepts described previously and combine it with the Web, preparing foundation for building Web-based IoT ecosystems.

We will start "building web-based IoT ecosystems" session by some motivation scenarios for the WoT 11, 5. Utilizing the RESTful web services, standards and best practices can bring harmony and interoperability among the IoT platforms and ecosystems. The session will highlight the use of semantics through RDFa, JSON-LD for IoT thing description and standardization efforts by W3C WoT Working Group (WG) ${ }^{6}$ The WoT WG activities cover those aspects that the WoT Interest Group (IG) believes are mature enough to progress to W3C Recommendations. The WoT activities included a task force on Thing Description (TD) that looked into semantic-based representation of things. Such TD has more granularity because of its representation in terms of events, properties and action. Another task force on Thing Discovery explored semantic-based discovery. The current progress in W3C WoT groups portrays the WoT as an abstraction layer on top of the IoT platforms 7 This section will also highlight benefits of utilizing the semantic web technologies and how WoT and Semantic Web concepts can be integrated in real-world applications.

These concepts will be delivered using examples with code snippets. We will present hands-on tips to start with the IoT application development immediately and demonstrate our current project - IoTSuite 14, 3, a toolkit for prototyping IoT applications ${ }^{8}$

\subsection{PART II: Semantic Web of Things Plat- forms}

Duration: 30 mins

Presenter: Amelie Gyrard.

While the previous session outlines the benefits of integrating semantic web technologies to IoT and WoT, this session dives deeper into semantics. It explains the way to semantically annotate data produced by IoT devices by us-

6ttps://www.w3.org/WoT/WG/

7 http://w3c.github.io/wot/charters/

wot-white-paper-2016.html

chttps://github.com/pankeshlinux/IoTSuite 
ing the W3C Semantic Sensor Networks (SSN) ontology ${ }^{9}$ and the Machine-to-Machine Measurement (M3) ontology to unify terms to describe sensor data. The M3 Framework has been designed to assist developers in developing semantics-based WoT applications 8. M3 enables enriching WoT data with meaningful information using logic-based inference engine (e.g., deducing hot from an outdoor temperature) but also enables combining IoT application domains 7 . The design of the M3 semantic engine is flexible and generic enough to be deployed at a cloud ${ }^{10}$ or an M2M Gateway or even an Android powered device.

The M3 semantic engine has been integrated within the FIESTA-IoT prototyp ${ }^{11}$ which comprises a set of tools to register and discover testbeds and experiments to later run the experiment to provide smart IoT applications to end-users. Sensor data produced by testbeds (e.g., Santander a smart city in Spain) is exploited and combined with other data coming from others testbeds. The FIESTA-IoT EU project ${ }^{12}$ ensures semantic interoperability of IoT data, testbeds and experiments/applications. The real FIESTAIoT platform ${ }^{13}$ is under development.

The M3 semantic engine will be explained in this context and the hands-on session will enable to use it in practice. Slides will be presented and followed by demos recorded illustrating the M3 framework and FIESTA-IoT project.

\subsection{PART III: Smart City Demos}

\section{Duration: 30 mins}

Presenter: Muhammad Intizar Ali.

Realizing the true potential of semantic technologies, various IoT frameworks have been proposed which address the data interoperability issues using the semantic Web technologies and standards 2]. In this session, we will demonstrate practical scenarios designed in the context of smart cities projects 2 , which leverage the benefits of semantic interoperability and provide real-time data analytics solutions for large-scale IoT data streams 15 . We will showcase several example applications using real-time data analytics and decision support systems. These applications (Smart City Interactive Dashboard, Multi-modal Context-aware Route Planner and Parking Space Finder) are developed for the City of Aarhus, Denmark using the CityPulse Framework 4. 1]. All demonstrated applications process real-time IoT data streams and perform real-time data analytics using semantic technologies and user-centric decision support. CityPulse is an EU project aiming at analyzing data provided by smart cites (such as Aarhus in Denmark ) with a focus on scalability to process large voume of streaming data. CityPulse develops semantics-based IoT applications. Slides will be presented and followed by demos. This session will also discuss few open challenges and research questions for WoT community [10, 9].

\subsection{PART IV: SWoT in Practice}

Duration: 60 mins

Presenters: Amelie Gyrard, Pankesh Patel, Muham-

\footnotetext{
$9 \longdiv { \text { https://www.w3.org/TR/vocab-ssn/ } }$

10 http://sensormeasurement .appspot.com/

11 http://fiesta-iot-tools.appspot.com/

12 http://fiesta-iot.eu/

13 https://platform-dev.fiesta-iot.eu/
}

mad Intizar Ali.

The last section is for practitioning the different tools presented previously: IoT tools, WoT tools, SWoT tools and data analytics tools to be able to design semantics-based IoT/WoT applications:

1. Running IoTSuite.

2. Running M3 framework on the Cloud and embedded on Android-powered devices.

3. Data Analytics with CityPulse.

4. Semantic interoperability with FIESTA-IoT.

The tutorial will wrap up with a focused discussion on specific learning of the audience and open questions.

\section{ORGANISERS}

Four presenters are involved in this tutorial as follows.

\subsection{Presenter: Amelie Gyrard}

Institute: Univ Lyon, MINES Saint-Etienne, CNRS, Laboratoire Hubert Curien, Saint-Etienne, France

Email: amelie.gyrard@emse.fr

Homepage:

http://sensormeasurement . appspot. com/?p=AmelieGyrard

She is a post-doc researcher at Ecole des Mines de SaintEtienne, France, working within the Connected Intelligence - Knowledge Representation and Reasoning team. Previously, she was a post-doc at Insight Center for Data Analytics, National University of Galway and actively working in the scientific development and coordination of the FIESTAIoT (Federated Interoperable Semantic IoT/Cloud Testbeds and Applications) EU H2020 project. Her research interests are on Software engineering for Semantic Web of Things and Internet of Things (IoT), semantic web best practices and methodologies, ontology engineering, reasoning and interoperability of IoT data. She holds a Ph.D. from Eurecom since April 2015 where she designed and implemented the Machine-to-Machine Measurement (M3) framework 6]. The title of her dissertation is "Designing Cross-Domain Semantic Web of Things Applications". She also disseminated her work in standardizations such as ETSI M2M, oneM2M, and W3C Web of Things.

\subsection{Presenter: Pankesh Patel}

Institute: ABB Corporate Research, India

Email: pankesh.patel@in.abb.com

Homepage: https://in.linkedin.com/in/pankeshpatel He is serving ABB Corporate Research-Bangalore, India as a Research Scientist. He focuses in building software development methodologies and tools to easily develop applications in the cross-section of Software Engineering, Cyber-Physical Systems, and Internet of Things. He regularly serves various academic events as PC and invited/keynote speaker. He obtained his Ph.D. from the University of Paris VI (UPMC) and INRIA (French National Institute for Research in Computer Science and Automation) Paris, France.

\subsection{Presenter: Soumya Kanti Datta}

Institute: EURECOM, Sophia Antipolis, France

Email: dattas@eurecom.fr

Web page: http://iot.eurecom.fr/

Soumya is a Research Engineer in EURECOM, France. He is working on French national and EU H2020 research projects 
and technology transfer to the IoT industry. His research focuses on innovation, standardization and development of next-generation technologies in the IoT, WoT and Smart Cities. He is an active member of IEEE Consumer Electronics (CE) Society. He leads the activities of IEEE CE Society Future Directions Team on IoT. He has published more than 55 research papers in top ACM and IEEE Conferences, Magazines and Journals. Soumya has served several IEEE conferences in many capacities. Currently he is involved in oneM2M, W3C Web of Things Working Group and contributing to their standard development activities. He obtained an M.Sc in Communications and Computer Security from Telecom ParisTech (EURECOM), France.

\subsection{Presenter: Muhammad Intizar Ali}

Institute: Insight Center for Data Analytics, National University of Ireland, Galway

Email: ali.intizar@insight-centre.org

Homepage: http://www.intizarali.org/

Dr. Muhammad Intizar Ali is an Adjunct Lecturer, Research Fellow and Research Unit Leader of Reasoning, Querying, and IoT Data Analytics Unit at Insight Centre for Data Analytics, National University of Ireland, Galway. His research interests include Semantic Web, Data Integration, Internet of Things (IoT), Linked Data, Federated Query Processing, Stream Query Processing and Optimal Query Processing over large scale distributed data sources. He is actively involved in various EU funded and industry-funded projects aimed at providing IoT enabled adaptive intelligence for smart city applications and smart enterprise communication systems. He is serving as a PC member of various journals, international conferences and workshops. He is also actively participating in W3C efforts for standardization in RDF Stream Processing Community Group. Dr. Ali obtained his Ph.D. (with distinction) from Vienna University of Technology, Austria in 2011.

\section{ACKNOWLEDGMENTS}

This work is partially funded by a bilateral research convention with ENGIE Research \& Development, the ANR 14-CE24-0029 OpenSensingCity project ${ }^{14}$ and institutional collaboration supported by the Horizon 2020 Programme European project "Federated Interoperable Semantic IoT/cloud Testbeds and Applications" (FIESTA-IoT) from the European Union with the Grant Agreement No. CNECTICT-643943, Science Foundation Ireland (SFI) under grant No. SFI/12/RC/228, EU FP7 CityPulse Project under grant No.603095 $5^{15}$ and French ANR project DataTweet ${ }^{16}$

\section{REFERENCES}

[1] M. I. Ali, F. Gao, and A. Mileo. CityBench: A Configurable Benchmark to Evaluate RSP Engines Using Smart City Datasets. In The Semantic Web-ISWC 2015, pages 374-389. Springer, 2015.

[2] P. Barnaghi, R. Tönjes, J. Höller, M. Hauswirth, A. Sheth, and P. Anantharam. Citypulse: Real-time iot stream processing and large-scale data analytics for

\footnotetext{
14 http://opensensingcity.emse.fr

15 http://www.ict-citypulse.eu

${ }^{16}$ http://www.agence-nationale-recherche.fr/?Projet= ANR-13-INFR-0008
}

smart city applications. In European Semantic Web Conference (ESWC), 2014.

[3] S. Chauhan, P. Patel, A. Sureka, F. C. Delicato, and S. Chaudhary. Demonstration Abstract: IoTSuite - A Framework to Design, Implement, and Deploy IoT Applications. In 15th ACM/IEEE International Conference on Information Processing in Sensor Networks (IPSN), April 2016.

[4] F. Gao, M. I. Ali, and A. Mileo. Semantic discovery and integration of urban data streams. In Proceedings of the Fifth International Conference on Semantics for Smarter Cities-Volume 1280, pages 15-30. CEUR-WS. org, 2014.

[5] D. Guinard, V. Trifa, and E. Wilde. A resource oriented architecture for the web of things. In Internet of Things (IOT), 2010, pages 1-8, Nov 2010.

6] A. Gyrard. Designing Cross-Domain Semantic Web of Things Applications. PhD thesis, Telecom ParisTech, Eurecom, April 2015.

[7] A. Gyrard, C. Bonnet, K. Boudaoud, and M. Serrano. Assisting IoT Projects and Developers in Designing Interoperable Semantic Web of Things Applications. In IEEE International Conference on Internet of Things 2015 (iThings), 2015.

[8] A. Gyrard, S. K. Datta, C. Bonnet, and K. Boudaoud. Cross-domain Internet of Things application development: M3 framework and evaluation. In FICLOUD 2015, 3rd International Conference on Future Internet of Things and Cloud, August 24-26, Rome, Italy, 2015.

[9] A. Kamilaris and M. I. Ali. Do Web of Things Truly Follow the Web of Things? . In IEEE World Forum for Io T. 2016.

[10] A. Kamilaris, S. Yussuf, and M. I. Ali. WOTS2E: A Search Engine for a Semantic Web of Things. In IEEE World Forum for Io T. 2016.

[11] F. Paganelli, S. Turchi, and D. Giuli. A web of things framework for restful applications and its experimentation in a smart city. IEEE Systems Journal, PP(99):1-12, 2014.

[12] P. Patel, A. Kattepur, D. Cassou, and G. Bouloukakis. Evaluating the Ease of Application Development for the Internet of Things. Technical report, Feb. 2013.

[13] P. Patel, A. Pathak, T. Teixeira, and V. Issarny. Towards application development for the internet of things. In Proceedings of the 8th Middleware Doctoral Symposium, page 5. ACM, 2011.

[14] D. Soukaras, P. Patel, H. Song, and S. Chaudhary. IoTSuite: a ToolSuite for prototyping internet of things applications. In The 4th International Workshop on Computing and Networking for Internet of Things (ComNet-IoT), co-located with 16th International Conference on Distributed Computing and Networking (ICDCN), 2015.

[15] R. Tonjes, M. I. Ali, P. Barnaghi, S. Ganea, F. Ganz, M. Haushwirth, B. Kjargaard, D. Kumper, A. Mileo, S. Nechifor, et al. Real time iot stream processing and large-scale data analytics for smart city applications. In European Conference on Networks and Communications, 2014. 